\title{
ON THE SOLUTIONS OF NONLINEAR INITIAL-BOUNDARY VALUE PROBLEMS
}

\author{
VLADIMÍR ĎURIKOVIČ AND MONIKA ĎURIKOVIČOVÁ
}

Received 27 September 2002

We deal with the general initial-boundary value problem for a second-order nonlinear nonstationary evolution equation. The associated operator equation is studied by the Fredholm and Nemitskii operator theory. Under local Hölder conditions for the nonlinear member, we observe quantitative and qualitative properties of the set of solutions of the given problem. These results can be applied to different mechanical and natural science models.

\section{Introduction}

The generic properties of solutions of the second-order ordinary differential equations were studied by Brüll and Mawhin in [2], Mawhin in [7], and by Šeda in [8]. Such questions were solved for nonlinear diffusional-type problems with the Dirichlet-, Neumann-, and Newton-type conditions in $[5,6]$.

In this paper, we study the set structure of classic solutions, bifurcation points and the surjectivity of an associated operator to a general second-order nonlinear evolution problem by the Fredholm operator theory. The present results allow us to search the generic properties of nonparabolic models which describe mechanical, physical, reactiondiffusion, and ecology processes.

\section{The formulation of the problem and basic notions}

Throughout this paper, we assume that the set $\Omega \subset \mathbb{R}^{n}$ for $n \in \mathbb{N}$ is a bounded domain with the sufficiently smooth boundary $\partial \Omega$. The real number $T$ is positive and $Q:=(0, T] \times \Omega, \Gamma:=(0, T] \times \partial \Omega$.

We use the notation $D_{t}$ for $\partial / \partial t, D_{i}$ for $\partial / \partial x_{i}, D_{i j}$ for $\partial^{2} / \partial x_{i} \partial x_{j}$, where $i, j=1, \ldots, n$, and $D_{0} u$ for $u$. The symbol cl $M$ means the closure of a set $M$ in $\mathbb{R}^{n}$.

We consider the nonlinear differential equation (possibly of a nonparabolic type)

$$
D_{t} u-A\left(t, x, D_{x}\right) u+f\left(t, x, u, D_{1} u, \ldots, D_{n} u\right)=g(t, x)
$$


for $(t, x) \in Q$, where the coefficients $a_{i j}, a_{i}, a_{0}$, for $i, j=1, \ldots, n$, of the second-order linear operator

$$
A\left(t, x, D_{x}\right) u=\sum_{i, j=1}^{n} a_{i j}(t, x) D_{i j} u+\sum_{i=1}^{n} a_{i}(t, x) D_{i} u+a_{0}(t, x) u
$$

are continuous functions from the space $C(\mathrm{cl} Q, \mathbb{R})$. The function $f$ is from the space $C\left(\operatorname{cl} Q \times \mathbb{R}^{n+1}, \mathbb{R}\right)$ and $g \in C(\operatorname{cl} Q, \mathbb{R})$.

Together with (2.1), we consider the following general homogeneous boundary condition:

$$
\left.B_{3}\left(t, x, D_{x}\right) u\right|_{\Gamma}:=\sum_{i=1}^{n} b_{i}(t, x) D_{i} u+\left.b_{0}(t, x) u\right|_{\Gamma}=0,
$$

where the coefficients $b_{i}$, for $i=1, \ldots, n$, and $b_{0}$ are continuous functions from $C(\operatorname{cl} \Gamma, \mathbb{R})$.

Furthermore, we require for the solution of (2.1) to satisfy the homogeneous initial condition

$$
\left.u\right|_{t=0}=0 \quad \text { on } \mathrm{cl} \Omega
$$

Remark 2.1. In the case where $b_{i}=0$, for $i=1, \ldots, n$, and $b_{0}=1$ in (2.3), we get the Dirichlet problem studied in [5].

If we consider the vector function $v:=\left(0, v_{1}, \ldots, v_{n}\right): \operatorname{cl} \Gamma \rightarrow \mathbb{R}^{n+1}$ and the value $v(t, x)$ which means the unit inner normal vector to $\mathrm{cl} \Gamma$ at the point $(t, x) \in \mathrm{cl} \Gamma$ and we let $b_{i}=v_{i}$ for $i=1, \ldots, n$ on $\mathrm{cl} \Gamma$, then problem (2.1), (2.3), (2.4) represents the Newton or Neuman problem investigated in [6].

Our considerations are concerned with a broad class of nonparabolic operators.

In the following definitions, we will use the notations

$$
\begin{gathered}
\langle u\rangle_{t, \mu, Q}^{s}:=\sup _{\substack{(t, x),(s, x) \in \mathrm{cl} Q \\
t \neq s}} \frac{|u(t, x)-u(s, x)|}{|t-s|^{\mu}}, \\
\langle u\rangle_{x, v, Q}^{y}:=\sup _{\substack{(t, x),(t, y) \in \mathrm{cl} Q \\
x \neq y}} \frac{|u(t, x)-u(t, y)|}{|x-y|^{v}}, \\
\langle f\rangle_{t, x, u}^{s, y, v}:=\left|f\left(t, x, u_{0}, u_{1}, \ldots, u_{n}\right)-f\left(s, y, v_{0}, v_{1}, \ldots, v_{n}\right)\right|, \\
\langle f\rangle_{t, x, u(t, x)}^{s, y, v(s, y)}:=\mid f\left[t, x, u(t, x), D_{1} u(t, x), \ldots, D_{n} u(t, x)\right], \\
\quad-f\left[s, y, v(s, y), D_{1} v(s, y), \ldots, D_{n} v(s, y)\right] \mid,
\end{gathered}
$$

where $x=\left(x_{1}, \ldots, x_{n}\right), y=\left(y_{1}, \ldots, y_{n}\right)$ are from $\mathbb{R}^{n},|x-y|=\left[\sum_{i=1}^{n}\left(x_{i}-y_{i}\right)^{2}\right]^{1 / 2}$, and $\mu, \nu \in \mathbb{R}$.

We will need the following Hölder spaces (see [4, page 147]). 
Definition 2.2. Let $\alpha \in(0,1)$.

(1) By the symbol $C_{t, x}^{(1+\alpha) / 2,1+\alpha}(\mathrm{cl} Q, \mathbb{R})$ we denote the vector space of continuous functions $u: \operatorname{cl} Q \rightarrow \mathbb{R}$ which have continuous derivatives $D_{i} u$ for $i=1, \ldots, n$ on $\mathrm{cl} Q$, and the norm

$$
\begin{aligned}
\|u\|_{(1+\alpha) / 2,1+\alpha, Q}:= & \sum_{i=0}^{n} \sup _{(t, x) \in \mathrm{cl} Q}\left|D_{i} u(t, x)\right|+\langle u\rangle_{t,(1+\alpha) / 2, Q}^{s} \\
& +\sum_{i=1}^{n}\left\langle D_{i} u\right\rangle_{t, \alpha / 2, Q}^{s}+\sum_{i=1}^{n}\left\langle D_{i} u\right\rangle_{x, \alpha / 2, Q}^{y}
\end{aligned}
$$

is finite.

(2) The symbol $C_{(t, x)}^{(2+\alpha) / 2,2+\alpha}(\mathrm{cl} Q, \mathbb{R})$ means the vector space of continuous functions $u$ : $\mathrm{cl} Q \rightarrow \mathbb{R}$ for which there exist continuous derivatives $D_{t} u, D_{i} u, D_{i j} u$ on $\operatorname{cl} Q, i, j=1, \ldots, n$, and the norm

$$
\begin{aligned}
\|u\|_{(2+\alpha) / 2,2+\alpha, Q}= & \sum_{i=0}^{n} \sup _{(t, x) \in \mathrm{cl} Q}\left|D_{i} u(t, x)\right|+\sup _{(t, x) \in \mathrm{cl} Q}\left|D_{t} u(t, x)\right| \\
& +\sum_{i, j=1}^{n} \sup _{(t, x) \in \mathrm{cl} Q}\left|D_{i j} u(t, x)\right|+\sum_{i=1}^{n}\left\langle D_{i} u\right\rangle_{t,(1+\alpha) / 2, Q}^{s}+\left\langle D_{t} u\right\rangle_{t, \alpha / 2, Q}^{s} \\
& +\sum_{i, j=1}^{n}\left\langle D_{i j} u\right\rangle_{t, \alpha / 2, Q}^{s}+\left\langle D_{t} u\right\rangle_{x, \alpha, Q}^{y}+\sum_{i, j=1}^{n}\left\langle D_{i j} u\right\rangle_{x, \alpha, Q}^{y}
\end{aligned}
$$

is finite.

(3) The symbol $C_{t, x}^{(3+\alpha) / 2,3+\alpha}(\mathrm{cl}, \mathbb{R})$ means the vector space of continuous functions $u: \operatorname{cl} Q \rightarrow \mathbb{R}$ for which the derivatives $D_{t}, D_{i} u, D_{t} D_{i} u, D_{i j} u, D_{i j k} u, i, j, k=1, \ldots, n$, are continuous on $\mathrm{cl} Q$, and the norm

$$
\begin{aligned}
\|u\|_{(3+\alpha) / 2,3+\alpha, Q}:= & \sum_{i=0}^{n} \sup _{(t, x) \in \mathrm{cl} Q}\left|D_{i} u(t, x)\right|+\sum_{i, j=1}^{n} \sup _{(t, x) \in \mathrm{cl} Q}\left|D_{i j} u(t, x)\right| \\
& +\sum_{i=0}^{n} \sup _{(t, x) \in \mathrm{cl} Q}\left|D_{t} D_{i} u(t, x)\right|+\sum_{i, j, k=1}^{n} \sup _{(t, x) \in \mathrm{cl} Q}\left|D_{i j k} u(t, x)\right| \\
& +\left\langle D_{t} u\right\rangle_{t,(1+\alpha) / 2, Q}^{s}+\sum_{i, j=1}^{n}\left\langle D_{i j} u\right\rangle_{t,(1+\alpha) / 2, Q}^{s} \\
& +\sum_{i=1}^{n}\left\langle D_{t} D_{i} u\right\rangle_{t, \alpha / 2, Q}^{s}+\sum_{i, j, k=1}^{n}\left\langle D_{i j k} u\right\rangle_{t, \alpha / 2, Q}^{s} \\
& +\sum_{i=1}^{n}\left\langle D_{t} D_{i} u\right\rangle_{x, \alpha, Q}^{y}+\sum_{i, j, k=1}^{n}\left\langle D_{i j k} u\right\rangle_{x, \alpha, Q}^{y}
\end{aligned}
$$

is finite.

The above-defined norm spaces are Banach ones. 
Definition 2.3 (the smoothness condition $\left(S_{3}^{1+\alpha}\right)$ ). Let $\alpha \in(0,1)$. The differential operators $A\left(t, x, D_{x}\right)$ from (2.1) and $B_{3}\left(t, x, D_{x}\right)$ from (2.3) satisfy the smoothness condition $\left(\mathrm{S}_{3}^{1+\alpha}\right)$ if, respectively,

(i) the coefficients $a_{i j}, a_{i}, a_{0}$ from (2.1), for $i, j=1, \ldots, n$, belong to the space $C_{t, x}^{(1+\alpha) / 2,1+\alpha}(\mathrm{cl} Q, \mathbb{R})$ and $\partial \Omega \in C^{3+\alpha}$,

(ii) the coefficients $b_{i}$ from $(2.3)$, for $i=1, \ldots, n$, belong to the space $C_{t, x}^{(2+\alpha) / 2,2+\alpha}(\mathrm{cl} \Gamma, \mathbb{R})$.

Definition 2.4 (the complementary condition (C)). If at least one of the coefficients $b_{i}$, for $i=1, \ldots, n$, of the differential operator $B_{3}\left(t, x, D_{x}\right)$ in $(2.3)$ is not zero, then $B_{3}\left(t, x, D_{x}\right)$ satisfies the complementary condition $(C)$.

Now, we are prepared to formulate hypotheses for deriving fundamental lemmas.

Definition 2.5. (1) Fredholm conditions.

(A1) Consider the operator $A_{3}: X_{3} \rightarrow Y_{3}$, where

$$
A_{3} u=D_{t} u-A\left(t, x, D_{x}\right) u, \quad u \in X_{3},
$$

and the operators $A\left(t, x, D_{x}\right)$ and $B_{3}\left(t, x, D_{x}\right)$ satisfy the smoothness condition $\left(\mathrm{S}_{3}^{1+\alpha}\right)$ for $\alpha \in(0,1)$ and the complementary condition $(\mathrm{C})$. Here, we consider the vector spaces

$$
\begin{aligned}
& D\left(A_{3}\right):=\left\{u \in C_{t, x}^{(3+\alpha) / 2,3+\alpha}(\operatorname{cl} Q, \mathbb{R}) ;\left.B_{3}\left(t, x, D_{x}\right) u\right|_{\Gamma}=0,\left.u\right|_{t=0}(x)=0 \text { for } x \in \operatorname{cl} Q\right\} \\
& H\left(A_{3}\right):=\left\{v \in C_{t, x}^{(1+\alpha) / 2,1+\alpha}(\operatorname{cl} Q, \mathbb{R}) ;\left.B_{3}\left(t, x, D_{x}\right) v(t, x)\right|_{t=0, x \in \partial \Omega}=0\right\}
\end{aligned}
$$

and Banach subspaces (of the given Hölder spaces)

$$
\begin{aligned}
& X_{3}=\left(D\left(A_{3}\right),\|\cdot\|_{(3+\alpha) / 2,3+\alpha, Q}\right), \\
& Y_{3}=\left(H\left(A_{3}\right),\|\cdot\|_{(1+\alpha) / 2,1+\alpha, Q}\right) .
\end{aligned}
$$

(A2) There is a second-order linear homeomorphism $C_{3}: X_{3} \rightarrow Y_{3}$ with

$$
C_{3} u=D_{t} u-C\left(t, x, D_{x}\right) u, \quad u \in X_{3}
$$

where

$$
C\left(t, x, D_{x}\right) u=\sum_{i, j=1}^{n} c_{i j}(t, x) D_{i j} u+\sum_{i=1}^{n} c_{i}(t, x) D_{i} u+c_{0}(t, x) u
$$

satisfying the smoothness condition $\left(S_{3}^{1+\alpha}\right)$. The operator $C_{3}$ is not necessarily a parabolic one.

(2) Local Hölder and compatibility conditions. 
Let $f:=f\left(t, x, u_{0}, u_{1}, \ldots, u_{n}\right): c l Q \times \mathbb{R}^{n+1} \rightarrow \mathbb{R}, \alpha \in(0,1)$, and let $p, q$, $p_{r}$, for $r=$ $0,1, \ldots, n$ be nonnegative constants. Here, $D$ represents any compact subset of $(\mathrm{cl} Q) \times$ $\mathbb{R}^{n+1}$. For $f$, we need the following assumptions:

(B1) let $f \in C^{1}\left(\mathrm{cl} Q \times \mathbb{R}^{n+1}, \mathbb{R}\right)$ and let the first derivatives $\partial f / \partial x_{i}, \partial f / \partial u_{j}$ be locally Hölder continuous on $\mathrm{cl} Q \times \mathbb{R}^{n+1}$ such that

$$
\begin{aligned}
\left\langle\frac{\partial f}{\partial x_{i}}\right\rangle_{t, x, u}^{s, y, v} & \leq p|t-s|^{\alpha / 2}+q|x-y|^{\alpha}+\sum_{r=0}^{n} p_{r}\left|u_{r}-v_{r}\right|, \\
\left\langle\frac{\partial f}{\partial u_{j}}\right\rangle_{t, x, u}^{s, y, v} & \leq p|t-s|^{\alpha / 2}+q|x-y|^{\alpha}+\sum_{r=0}^{n} p_{r}\left|u_{r}-v_{r}\right|,
\end{aligned}
$$

for $i=1, \ldots, n, j=0,1, \ldots, n$, and any $D$;

(B2) let $f \in C^{3}\left(\mathrm{cl} Q \times \mathbb{R}^{n+1}, \mathbb{R}\right)$ and let the local growth conditions for the third derivatives of $f$ hold on any $D$ :

$$
\begin{aligned}
&\left\langle\frac{\partial^{3} f}{\partial \tau \partial x_{i} \partial u_{j}}\right\rangle_{t, x, u}^{t, x, v} \leq \sum_{s=0}^{n} p_{s}\left|u_{s}-v_{s}\right|^{\beta_{s}}, \\
&\left\langle\frac{\partial^{3} f}{\partial \tau \partial u_{j} \partial u_{k}}\right\rangle_{t, x, u}^{t, x, v} \leq \sum_{s=0}^{n} p_{s}\left|u_{s}-v_{s}\right|^{\beta_{s}}, \\
&\left\langle\frac{\partial^{3} f}{\partial x_{i} \partial x_{l} \partial u_{j}}\right\rangle_{t, x, u}^{t, x, v} \leq \sum_{s=0}^{n} p_{s}\left|u_{s}-v_{s}\right|^{\beta_{s}}, \\
&\left\langle\frac{\partial^{3} f}{\partial x_{i} \partial u_{j} \partial u_{k}}\right\rangle_{t, x, u}^{t, x, v} \leq \sum_{s=0}^{n} p_{s}\left|u_{s}-v_{s}\right|^{\beta_{s}}, \\
&\left\langle\frac{\partial^{3} f}{\partial u_{j} \partial u_{k} \partial u_{r}}\right\rangle_{t, x, u}^{t, x, v}, \leq \sum_{s=0}^{n} p_{s}\left|u_{s}-v_{s}\right|^{\beta_{s}},
\end{aligned}
$$

where $\beta_{s}>0$ for $s=0,1, \ldots, n$ and $i, l=1, \ldots, n ; j, k, r=0,1, \ldots, n$;

(B3) the equality of compatibility

$$
\sum_{i=1}^{n} b_{i}(t, x) D_{i} f(t, x, 0, \ldots, 0)+\left.b_{0}(t, x) f(t, x, 0, \ldots, 0)\right|_{t=0, x \in \partial \Omega}=0
$$

holds.

(3) Almost coercive condition.

Let, for any bounded set $M_{3} \subset Y_{3}$, there exist a number $K>0$ such that for all solutions $u \in X_{3}$ of problem (2.1), (2.3), (2.4) with the right-hand sides $g \in M_{3}$, the following alternative holds: 
412 Solutions of nonlinear initial-boundary value problems

(C1) either

$\left(\alpha_{1}\right)\|u\|_{(1+\alpha) / 2,1+\alpha, Q} \leq K, f:=f\left(t, x, u_{0}\right): c l Q \times \mathbb{R} \rightarrow \mathbb{R}$, and the coefficients of the operators $A_{3}$ and $C_{3}$ (see (2.1) and (A2)) satisfy the equations

$$
a_{i j}=c_{i j}, \quad a_{i}=c_{i}, \quad \text { for } i, j=1, \ldots, n, \quad a_{0} \neq c_{0} \quad \text { on } \mathrm{cl} Q,
$$

or

$\left(\alpha_{2}\right)\|u\|_{(2+\alpha) / 2,2+\alpha, Q} \leq K, f:=f\left(t, x, u_{0}, u_{1}, \ldots, u_{n}\right): c l Q \times \mathbb{R}^{n+1} \rightarrow \mathbb{R}$, and the coefficients of the operators $A_{3}$ and $C_{3}$ satisfy the relations

$$
\begin{aligned}
& a_{i j}=c_{i j} \quad \text { for } i, j=1, \ldots, n, \quad a_{i} \neq c_{i} \text { for at least one } i=1, \ldots, n \\
& \text { on clQ. }
\end{aligned}
$$

Remark 2.6. (1) Especially, condition (A2) is satisfied for the diffusion operator

$$
C_{3} u=D_{t} u-\triangle u, \quad u \in X_{3}
$$

or for any uniformly parabolic operator $C_{3}$ with sufficiently smooth coefficients. However, the operator $C_{3}$ is not necessarily uniform parabolic.

(2) The local Hölder conditions in (B1) and (B2) admit sufficiently strong growths of $f$ in the last variables $u_{0}, u_{1}, \ldots, u_{n}$. For example, they include exponential and power-type growths.

Definition 2.7. (1) A couple $(u, g) \in X_{3} \times Y_{3}$ will be called the bifurcation point of the mixed problem (2.1), (2.3), (2.4) if $u$ is a solution of that mixed problem and there exists a sequence $\left\{g_{k}\right\} \subset Y_{3}$ such that $g_{k} \rightarrow g$ in $Y_{3}$ as $k \rightarrow \infty$, and problem (2.1), (2.3), (2.4) for $g=g_{k}$ has at least two different solutions $u_{k}, v_{k}$ for each $k \in \mathbb{N}$ and $u_{k} \rightarrow u, v_{k} \rightarrow u$ in $X_{3}$ as $k \rightarrow \infty$.

(2) The set of all solutions $u \in X_{3}$ of (2.1), (2.3), (2.4) (or the set of all functions $\left.g \in Y_{3}\right)$ such that $(u, g)$ is a bifurcation point of problem (2.1), (2.3), (2.4) will be called the domain of bifurcation (the bifurcation range) of that problem.

\section{Fundamental lemmas}

Lemma 3.1. Let conditions (A1) and (A2) hold (see Definition 2.5). Then,

(1) $\operatorname{dim} X_{3}=+\infty$;

(2) the operator $A_{3}: X_{3} \rightarrow Y_{3}$ is a linear bounded Fredholm operator of the zero index.

Proof. (1) To prove the first part of this lemma, we use the decomposition theorem from [9, page 139].

Let $X$ be a linear space and let $x^{*}: X \rightarrow \mathbb{R}$ be a linear functional on $X$ such that $x^{*} \neq 0$. Furthermore, let $M=\left\{x \in X ; x^{*}(x)=0\right\}$ and let $x_{0} \in X-M$. Then, every element $x \in X$ can be expressed by the formula

$$
x=\left[\frac{x^{*}(x)}{x^{*}\left(x_{0}\right)}\right] x_{0}+m, \quad m \in M,
$$

that is, there is a one-dimensional subspace $L_{1}$ of $X$ such that $X=L_{1} \oplus M$. 
If we now let

$$
M_{1}:=\left\{u \in C_{t, x}^{(3+\alpha) / 2,3+\alpha}(\operatorname{cl} Q, \mathbb{R})=: H^{3+\alpha} ;\left.B_{3}\left(t, x, D_{x}\right) u\right|_{\Gamma}=0\right\},
$$

which is the linear subspace of $H^{3+\alpha}$, then there exists a linear subspace $L_{1}$ of $H^{3+\alpha}$ with $\operatorname{dim} L_{1}=1$ such that $H^{3+\alpha}=L_{1} \oplus M_{1}$. Similarly, if we take $M_{2}:=\left\{u \in M_{1} ;\left.u\right|_{t=0}=\right.$ 0 on $\operatorname{cl} \Omega\}$, then there is a subspace $L_{2}$ of $M_{1}$ with $\operatorname{dim} L_{2}=1$ such that $M_{1}=L_{2} \oplus M_{2}$. Hence, we have $H^{3+\alpha}=L_{1} \oplus L_{2} \oplus D\left(A_{3}\right)$. Since $\operatorname{dim} H^{3+\alpha}=+\infty$, we get that $\operatorname{dim} X_{3}=+\infty$.

(2) (a) In the first step, we prove the boundedness of the linear operator $A_{3}$. To this end, we observe the norm $\left\|A_{3} u\right\|_{(1+\alpha) / 2,1+\alpha, Q}$ for $u \in D\left(A_{3}\right)$. From the assumption $\left(\mathrm{S}_{3}^{1+\alpha}\right)$ we get for $k=0,1, \ldots, n$,

$$
\sup _{(t, x) \in \mathrm{cl} Q}\left|D_{k} A_{3} u(t, x)\right| \leq K_{1}\|u\|_{(3+\alpha) / 2,3+\alpha, Q}, \quad K_{1}>0 .
$$

Applying again the smoothness assumption $\left(S_{3}^{1+\alpha}\right)$, the mean value theorem for the functions $u$ and $D_{i} u$, and the boundedness of $Q$, we obtain for the second member of the above-mentioned norm the following estimation:

$$
\begin{aligned}
\left\langle A_{3} u\right\rangle_{t,(1+\alpha) / 2, Q}^{s} & =\sup _{\substack{(t, x),(s, x) \in \mathrm{cl} Q \\
t \neq s}} \frac{\left|A_{3} u(t, x)-A_{3} u(s, x)\right|}{|t-s|^{(1+\alpha) / 2}} \\
& \leq K_{2}\|u\|_{(3+\alpha) / 2,3+\alpha, Q}, \quad K_{2}>0 .
\end{aligned}
$$

For the third member of the norm (2.6), we estimate for $k=1, \ldots, n$ as follows:

$$
\begin{aligned}
\left\langle D_{k} A_{3} u\right\rangle_{t, \alpha / 2, Q}^{s} & =\sup _{\substack{(t, x),(s, x) \in \mathrm{cl} Q \\
t \neq s}} \frac{\left|D_{k} A_{3} u(t, x)-D_{k} A_{3} u(s, x)\right|}{|t-s|^{\alpha / 2}} \\
& \leq K_{3}\|u\|_{(3+\alpha) / 2,3+\alpha, Q}, \quad K_{3}>0 .
\end{aligned}
$$

An estimation of the last member in (2.6) for $A_{3} u$ is given by the following inequality for $k=1, \ldots, n$ :

$$
\begin{aligned}
\left\langle D_{k} A_{3} u\right\rangle_{x, \alpha / 2, Q}^{y} & =\sup _{\substack{(t, x),(t, y) \in \mathrm{cl} Q \\
x \neq y}} \frac{\left|D_{k} A_{3} u(t, x)-D_{k} A_{3} u(t, y)\right|}{|x-y|^{\alpha / 2}} \\
& \leq K_{4}\|u\|_{(3+\alpha) / 2,3+\alpha, Q}, \quad K_{4}>0 .
\end{aligned}
$$

From the estimations (3.3), (3.4), (3.5), and (3.6), we can conclude that

$$
\left\|A_{3} u\right\|_{Y_{3}}=\left\|A_{3} u\right\|_{(1+\alpha) / 2,1+\alpha, Q} \leq K\left(n, T, \alpha, \Omega, a_{i j}, a_{i}, a_{0}\right)\|u\|_{X_{3}} .
$$

(b) To prove that $A_{3}$ is a Fredholm operator with the zero index, we express it in the form

$$
A_{3} u=C_{3} u+\left[C\left(t, x, D_{x}\right)-A\left(t, x, D_{x}\right)\right] u=: C_{3} u+T_{3} u
$$


414 Solutions of nonlinear initial-boundary value problems

where $C_{3}: X_{3} \rightarrow Y_{3}$ is a linear homeomorphism and $C$ is the linear operator from (A2). By the decomposition Nikolskii theorem [10, page 233], it is sufficient to show that $T_{3}$ : $X_{3} \rightarrow Y_{3}$ is a linear completely continuous operator.

The complete continuity of $T_{3}$ can be proved by the Ascoli-Arzelá theorem (see [11, page 141]).

From $\left(S_{3}^{1+\alpha}\right)$, the uniform boundedness of the operator

$$
\begin{aligned}
T_{3} u= & \sum_{i, j=1}^{n}\left[c_{i j}(t, x)-a_{i j}(t, x)\right] D_{i j} u+\sum_{i=1}^{n}\left[c_{i}(t, x)-a_{i}(t, x)\right] D_{i} u \\
& +\left[c_{0}(t, x)-a_{0}(t, x)\right] u
\end{aligned}
$$

follows by the same way as the boundedness of the operator $A_{3}$ in the previous part (1). Thus, for all $u \in M \subset X_{3}$, where $M$ is a set bounded by the constant $K_{1}>0$, we obtain the estimate

$$
\left\|T_{3} u\right\|_{Y_{3}} \leq K\left(n, \alpha T, \Omega, a_{i j}, c_{i j}, a_{i}, c_{i}, a_{0}, c_{0}\right)\|u\|_{X_{3}} \leq K K_{1} .
$$

Using the smoothness condition of the operators $A$ and $C$, we get the inequalities

$$
\begin{aligned}
\left|T_{3} u(t, x)-T_{3} u(s, y)\right| \leq & \sum_{i, j=1}^{n}\left|\left[c_{i j}-a_{i j}\right](t, x)-\left[c_{i j}-a_{i j}\right](s, y)\right|\left|D_{i j} u(t, x)\right| \\
& +\sum_{i, j=1}^{n}\left|c_{i j}(s, y)-a_{i j}(s, y)\right|\left|D_{i j} u(t, x)-D_{i j} u(s, y)\right| \\
& +\sum_{i=1}^{n}\left|\left[c_{i}-a_{i}\right](t, x)-\left[c_{i}-a_{i}\right](s, y)\right|\left|D_{i} u(t, x)\right| \\
& +\sum_{i=1}^{n}\left|c_{i}(s, y)-a_{i}(s, y)\right|\left|D_{i} u(t, x)-D_{i} u(s, y)\right| \\
& +\left|\left[c_{0}-a_{0}\right](t, x)-\left[c_{0}-a_{0}\right](s, y)\right||u(t, x)| \\
& +\left|c_{0}(s, y)-a_{0}(s, y)\right||u(t, x)-u(s, y)| \\
\leq & 4 K_{1} K n^{2}\left[|t-s|^{\alpha / 2}+|x-y|^{\alpha}\right] \\
& +2 K_{1} K n\left[\left(|t-s|^{\alpha / 2}+|x-y|^{\alpha}\right)+\left(|t-s|^{(1+\alpha) / 2}+|x-y|\right)\right] \\
& +2 K_{1} K\left[\left(|t-s|^{\alpha / 2}+|x-y|^{\alpha}\right)+(|t-s|+|x-y|)\right]
\end{aligned}
$$

where $K_{1}, K$ are positive constants. Hence, the equicontinuity of $T_{3} M \subset Y_{3}$ follows. This finishes the proof of Lemma 3.1.

Lemma 3.1 implies the following alternative. 
COROLlary 3.2. Let L mean the set of all second-order linear differential operators

$$
A_{3}=D_{t}-A\left(t, x, D_{x}\right): X_{3} \longrightarrow C_{t, x}^{(1+\alpha) / 2,1+\alpha}(\mathrm{cl} Q, \mathbb{R})
$$

satisfying conditions $(C)$ and $\left(S_{2}^{1+\alpha}\right)$. Then, for each $A_{3} \in L$, the mixed homogeneous problem $A_{3} u=0$ on $Q,(2.3)$, and (2.4) has a nontrivial solution or any $A_{3} \in L$ is a linear bounded Fredholm operator of the zero-index mapping $X_{3}$ onto $Y_{3}$.

The following lemma establishes the complete continuity of the Nemitskii operator from the nonlinear part of (2.1).

Lemma 3.3. Let assumptions (B1) and (B3) be satisfied. Then the Nemitskii operator $N_{3}$ : $X_{3} \rightarrow Y_{3}$ defined by

$$
\left(N_{3} u\right)(t, x)=f\left[t, x, u(t, x), D_{1} u(t, x), \ldots, D_{n} u(t, x)\right]
$$

for $u \in X_{3}$ and $(t, x) \in \mathrm{cl} Q$ is completely continuous.

Proof. Let $M_{3} \subset X_{3}$ be a bounded set. By the Ascoli-Arzelá theorem, it is sufficient to show that the set $N_{3}\left(M_{3}\right)$ is uniformly bounded and equicontinuous. We will use assumption (B3) to prove the inclusion $N_{3}\left(M_{3}\right) \subset Y_{3}$.

Take $u \in M_{3}$. According to assumption (B1), we obtain the local boundedness of the function $f$ and of its derivatives $\partial f / \partial x_{i}$ on $(\operatorname{cl} Q) \times \mathbb{R}^{n+1}$ for $i=1, \ldots, n$. From this and from the equation

$$
D_{i}\left(N_{3} u\right)(t, x)=\left\{D_{i} f[\cdot]+\sum_{l=0}^{n} \frac{\partial f}{\partial u_{l}}[\cdot] D_{i} D_{l} u\right\}\left[\cdot, \cdot, u, D_{1} u, \ldots, D_{n} u\right](t, x)
$$

we have the estimation

$$
\sup _{(t, x) \in \mathrm{clQ}}\left|D_{i}\left(N_{3} u\right)(t, x)\right| \leq K_{1}
$$

for $i=0,1, \ldots, n$ with a positive sufficiently large constant $K_{1}$ not depending on $u \in M_{3}$.

Using the differentiability of $f$ and the mean value theorem in the variable $t$ for the difference of the derivatives of $u$, we can write

$$
\left\langle N_{3} u\right\rangle_{t,(1+\alpha) / 2, Q}^{s} \leq K_{1} .
$$

Similarly, by (2.14), we have

$$
\left\langle D_{i} N_{3} u\right\rangle_{t, \alpha / 2, Q}^{s} \leq K_{1}, \quad\left\langle D_{i} N_{3} u\right\rangle_{x, \alpha, Q}^{y} \leq K_{1},
$$

for $i=1, \ldots, n$ and $u \in M_{3}$. The previous estimations yield the inequality

$$
\left\|N_{3} u\right\|_{Y_{3}} \leq K_{1}
$$

for all $u \in M_{3}$. 
416 Solutions of nonlinear initial-boundary value problems

With respect to (B1), for any $u \in M_{3}$ and $(t, x),(s, y) \in \operatorname{cl} Q$ such that $|t-s|^{2}+\mid x-$ $\left.y\right|^{2}<\delta^{2}$ with a sufficiently small $\delta>0$, we have

$$
\left|N_{3} u(t, x)-N_{3} u(s, y)\right|<\epsilon, \quad \epsilon>0,
$$

which is the equicontinuity of $N_{3}\left(M_{3}\right)$. This finishes the proof of Lemma 3.3.

Lemma 3.4. Let assumptions (A1), (A2), (B1), (B3), and (C1) hold. Then the operator $F_{3}=A_{3}+N_{3}: X_{3} \rightarrow Y_{3}$ is coercive.

Proof. We need to prove that if the set $M_{3} \subset Y_{3}$ is bounded in $Y_{3}$, then the set of arguments $F_{3}^{-1}\left(M_{3}\right) \subset X_{3}$ is bounded in $X_{3}$.

In both cases $\left(\alpha_{1}\right)$ and $\left(\alpha_{2}\right)$, we get for all $u \in F_{3}^{-1}\left(M_{3}\right)$,

$$
\left\|N_{3} u\right\|_{(1+\alpha) / 2,1+\alpha, Q} \leq K_{1},
$$

where $K_{1}>0$ is a sufficiently large constant. Hence,

$$
\left\|A_{3} u\right\|_{Y_{3}} \leq K_{1}
$$

for any $u \in F_{3}^{-1}\left(M_{3}\right)$.

Hypothesis (A2) ensures the existence and uniqueness of the solution $u \in X_{3}$ of the linear equation

$$
C_{3} u=y
$$

and for any $y \in Y_{3}$,

$$
\|u\|_{X_{3}} \leq K_{1}\|y\|_{Y_{3}} .
$$

If we write

$$
\begin{aligned}
C_{3} u= & A_{3} u+\sum_{i, j=1}^{n}\left[a_{i j}(t, x)-c_{i j}(t, x)\right] D_{i j} u \\
& +\sum_{i=1}^{n}\left[a_{i}(t, x)-c_{i}(t, x)\right] D_{i} u+\left[a_{0}(t, x)-c_{0}(t, x)\right] u,
\end{aligned}
$$

then in both cases and for each $u \in F_{3}^{-1}\left(M_{3}\right)$, we obtain

$$
\|y\|_{Y_{3}} \leq\left\|C_{3} u\right\|_{Y_{3}} \leq K_{1}
$$

whence, by inequality (3.23), we can conclude that the operator $F_{3}$ is coercive.

LeMmA 3.5. Let the Nemitskii operator $N_{3}: X_{3} \rightarrow Y_{3}$ from (3.13) satisfy conditions (B2) and (B3). Then the operator $N_{3}$ is continuously Fréchet-differentiable, that is, $N_{3} \in C^{1}\left(X_{3}\right.$, $\left.Y_{3}\right)$ and it is completely continuous. 
Proof. From (B2), we obtain (B1) which implies by Lemma 3.3 the complete continuity of $N_{3}$. To obtain the first part of the assertion of this lemma, we need to prove that the Fréchet derivative $N_{3}^{\prime}: X_{3} \rightarrow L\left(X_{3}, Y_{3}\right)$ defined by the equation

$$
N_{3}^{\prime}(u) h(t, x)=\sum_{j=0}^{n} \frac{\partial f}{\partial u_{j}}\left(t, x, u(t, x), D_{1} u(t, x), \ldots, D_{n} u(t, x)\right] D_{j} h(t, x)
$$

for $u, h \in X_{3}$ is continuous on $X_{3}$. Thus, we must prove, for every $v \in X_{3}$, that

$$
\forall \epsilon>0 \exists \delta(\epsilon, v)>0, \quad \forall u \in X_{3},\|u-v\|_{X_{3}}<\delta: \sup _{h \in X_{3},\|h\|_{X_{3}} \leq 1}\left\|\left[N_{3}^{\prime}(u)-N_{3}^{\prime}(v)\right] h\right\|_{Y_{3}}<\epsilon .
$$

Using the norms (2.6), (2.8) and the estimation $\|u-v\|_{X_{3}}<\delta$, we have for the first term of (3.27) by the mean value theorem,

$$
\begin{aligned}
& \sum_{i=0}^{n} \sup _{(t, x) \in \mathrm{cl} Q}\left|D_{i}\left[N_{3}^{\prime}(u)-N_{3}^{\prime}(v)\right] h(t, x)\right| \\
& \leq \sum_{i, j=0}^{n} \sup _{(t, x) \in \mathrm{cl} Q}\left[\left\langle\frac{\partial^{2} f}{\partial x_{i} \partial u_{j}}\right\rangle_{t, x, u(t, x)}^{t, x, v(t, x)}\left|D_{j} h(t, x)\right|\right. \\
&+\sum_{k=0}^{n}\left\langle\frac{\partial^{2} f}{\partial u_{j} \partial u_{k}}\right\rangle_{t, x, u(t, x)}^{t, x, v(t, x)}\left|D_{i k} u\right| \cdot\left|D_{j} h\right|(t, x) \\
&+\sum_{k=0}^{n}\left|\frac{\partial^{2} f}{\partial u_{j} \partial u_{k}}(t, x, v(t, x), \ldots)\right|\left|D_{i k} u-D_{i k} v\right|\left|D_{j} h\right|(t, x) \\
&\left.+\left\langle\frac{\partial f}{\partial u_{j}}\right\rangle_{t, x, u(t, x)}^{t, x, v(t, x)}\left|D_{i j} h(t, x)\right|\right]<K \delta, \quad K>0 .
\end{aligned}
$$

For the second term of (3.27), we estimate as follows:

$$
\begin{aligned}
& \left\langle\left[N_{3}^{\prime}(u)-N_{3}^{\prime}(v)\right] h\right\rangle_{t,(1+\alpha) / 2, Q}^{s} \\
& \leq \sum_{j=0}^{n} \sup _{\mathrm{cl} Q, t \neq s}|t-s|^{-(1+\alpha) / 2}\left[\left|\int_{s}^{t} D_{\tau}\left\langle\frac{\partial f}{\partial u_{j}}\right\rangle_{\tau, x, u(\tau, x)}^{\tau, x, v(\tau, x)} d \tau\right|\left|D_{j} h(t, x)\right|\right. \\
& \left.+\left\langle\frac{\partial f}{\partial u_{j}}\right\rangle_{s, x, u(s, x)}^{s, x, v(s, x)}\left|\int_{s}^{t} D_{\tau} D_{j} h(\tau, x) d \tau\right|\right] \\
& \leq K \delta, \quad K>0 .
\end{aligned}
$$

Here, we have used the mean value theorem for $\partial^{2} f / \partial \tau \partial u_{j}, \partial^{2} f / \partial u_{j} \partial u_{k}$, and $\partial f / \partial u_{j}$ for $j, k=0,1, \ldots, n$. 
418 Solutions of nonlinear initial-boundary value problems

The third term of (3.27) gives by (2.15),

$$
\begin{aligned}
& \sum_{i=1}^{n}\left\langle D_{i}\left\{\left[N_{3}^{\prime}(u)-N_{3}^{\prime}(v)\right] h\right\}\right\rangle_{t, \alpha / 2, Q}^{s} \\
& \leq \sum_{i=1}^{n} \sum_{j=0}^{n} \sup _{\mathrm{cl} Q, t \neq s}|t-s|^{-\alpha / 2} \\
& \times\left\{\left|\int_{s}^{t} D_{\tau}\left\langle\frac{\partial^{2} f}{\partial x_{i} \partial u_{j}}\right\rangle_{\tau, x, u(\tau, x)}^{\tau, x, v(\tau, x)} d \tau\right|\left|D_{j} h(t, x)\right|\right. \\
& +\left\langle\frac{\partial^{2} f}{\partial x_{i} \partial u_{j}}\right\rangle_{s, x, u(s, x)}^{s, x, v(s, x)}\left|\int_{s}^{t} D_{\tau} D_{j} h(\tau, x) d \tau\right| \\
& +\sum_{k=0}^{n}\left[\left|\int_{s}^{t} D_{\tau}\left\langle\frac{\partial^{2} f}{\partial u_{j} \partial u_{k}}\right\rangle_{\tau, x, u(\tau, x)}^{\tau, x, v(\tau, x)} d \tau\right|\left|D_{i k} u\right|\left|D_{j} h\right|(t, x)\right. \\
& +\left|\int_{s}^{t} D_{\tau}\left[\frac{\partial^{2} f}{\partial u_{j} \partial u_{k}}(\tau, x, v, \ldots) d \tau\right]\right| \\
& \times\left|D_{i k} u(t, x)-D_{i k} v(t, x)\right|\left|D_{j} h(t, x)\right| \\
& +\left\langle\frac{\partial^{2} f}{\partial u_{j} \partial u_{k}}\right\rangle_{s, x, u(s, x)}^{s, x, v(s, x)}\left|D_{i k} u(t, x)-D_{i k} u(s, x)\right|\left|D_{j} h(t, x)\right| \\
& +\left|\frac{\partial^{2} f}{\partial u_{j} \partial u_{k}}(s, x, v, \ldots)\right| \\
& \times\left|D_{i k} u(t, x)-D_{i k} v(t, x)-\left[D_{i k} u(s, x)-D_{i k} v(s, x)\right]\right|\left|D_{j} h(t, x)\right| \\
& +\left\langle\frac{\partial^{2} f}{\partial u_{j} \partial u_{k}}\right\rangle_{s, x, u(s, x)}^{s, x, v(s, x)}\left|D_{i k} u(s, x)\right|\left|\int_{s}^{t} D_{\tau} D_{j} h(\tau, x) d \tau\right| \\
& +\left|\frac{\partial^{2} f}{\partial u_{j} \partial u_{k}}(s, x, v, \ldots)\right|\left|D_{i k} u(s, x)-D_{i k} v(s, x)\right| \\
& \times\left|\int_{s}^{t} D_{\tau} D_{j} h(\tau, x) d \tau\right| \\
& +\left|\int_{s}^{t} D_{\tau}\left\langle\frac{\partial f}{\partial u_{j}}\right\rangle_{\tau, x, u(\tau, x)}^{\tau, x, v(\tau, x)} d \tau\right|\left|D_{i j} h(t, x)\right| \\
& \left.\left.+\left\langle\frac{\partial f}{\partial u_{j}}\right\rangle_{s, x, u(s, x)}^{s, x, v(s, x)}\left|D_{i j} h(t, x)-D_{i j} h(s, x)\right|\right]\right\} \\
& \leq K\left(\sum_{s=0}^{n} \delta^{\beta_{s}}+\delta\right), \quad K>0 .
\end{aligned}
$$


Making the corresponding changes, the last term of (3.27), by condition (B2), gives the required estimation:

$$
\sum_{i=1}^{n}\left\langle D_{i}\left\{\left[N_{3}^{\prime}(u)-N_{3}^{\prime}(v)\right] h\right\}\right\rangle_{x, \alpha, Q}^{y} .
$$

This finishes the proof of Lemma 3.5.

\section{Generic properties for continuous operators}

On a mutual equivalence between the solution of the given initial-boundary value problem and an operator equation, we have the following lemma.

Lemma 4.1. Let $A_{3}: X_{3} \rightarrow Y_{3}$ be the linear operator from Lemma 3.1, let $N_{3}: X_{3} \rightarrow Y_{3}$ be the Nemitskii operator from Lemma 3.3, and let $F_{3}=A_{3}+N_{3}: X_{3} \rightarrow Y_{3}$. Then,

(1) the function $u \in X_{3}$ is a solution of the initial-boundary value problem (2.1), (2.3), (2.4) for $g \in Y_{3}$ if and only if $F_{3} u=g$;

(2) the couple $(u, g) \in X_{3} \times Y_{3}$ is the bifurcation point of the initial-boundary value problem (2.1), (2.3), (2.4) if and only if $F_{3}(u)=g$ and $u \in \Sigma$, where $\sum$ means the set of all points of $X_{3}$ at which $F_{3}$ is not locally invertible.

Proof. (1) The first equivalence directly follows from the definition of the operator $F_{3}$ and of the mixed problem (2.1), (2.3), (2.4).

(2) If $(u, g)$ is a bifurcation point of the mixed problem (2.1), (2.3), (2.4) and $u_{k}, v_{k}$, and $g_{k}$ for $k=1,2, \ldots$ have the same meaning as in Definition 2.7, then with respect to (1) we have $F_{3}(u)=g, F_{3}\left(u_{k}\right)=g_{k}=F_{3}\left(v_{k}\right)$. Thus, $F_{3}$ is not locally injective at $u$. Hence, $F_{3}$ is not locally invertible at $u$, that is, $u \in \Sigma$. Conversely, if $F_{3}$ is not locally invertible at $u$ and $F_{3}(u)=g$, then $F_{3}$ is not locally injective at $u$. Indirectly, from Definition 2.7, we see that the couple $(u, g)$ is a bifurcation point of $(2.1),(2.3),(2.4)$.

LEMMA 4.2. Let

(i) the operator $A\left(t, x, D_{x}\right) \neq 0$ from (2.1) and the operator $B_{3}\left(t, x, D_{x}\right)$ from (2.3) satisfy the smoothness condition $\left(\mathrm{S}_{3}^{1+\alpha}\right)$;

(ii) the nonlinear part $f$ of $(2.1)$ belong to $C\left(\mathrm{cl} Q \times \mathbb{R}^{n+1}, \mathbb{R}\right)$;

(iii) the operator $A_{3}+N_{3}: X_{3} \rightarrow Y_{3}$ be nonconstant.

Then, for any compact set of the right-hand sides $g \in Y_{3}$ from (2.1), the set of all solutions of problem (2.1), (2.3), (2.4) is compact (possibly empty).

Proof. Following the proof of Lemma 3.1, we see that $\operatorname{dim} X_{3}=+\infty$ and the linear operator $A_{3}: X_{3} \rightarrow Y_{3}$ is continuous and accordingly closed. From hypothesis (ii) the Nemitskii operator $N_{3}: X_{3} \rightarrow Y_{3}$ given in (4.9) is closed too. By [8, Proposition 2.1], the operator $F_{3}=A_{3}+N_{3}: X_{3} \rightarrow Y_{3}$ is proper, and with respect to Lemma 4.1 we get our assertion.

THEOREM 4.3. Under assumptions (A1), (A2) and (B1), (B3), the following statements hold for problem (2.1), (2.3), (2.4): 
420 Solutions of nonlinear initial-boundary value problems

(a) the operator $F_{3}=A_{3}+N_{3}: X_{3} \rightarrow Y_{3}$ is continuous;

(b) for any compact set of the right-hand sides $g \in Y_{3}$ from (2.1), the corresponding set of all solutions is a countable union of compact sets;

(c) for $u_{0} \in X_{3}$, there exist neighborhoods $U\left(u_{0}\right)$ of $u_{0}$ and $U\left(F_{3}\left(u_{0}\right)\right)$ of $F_{3}\left(u_{0}\right) \in Y_{3}$ such that for each $g \in U\left(F_{3}\left(u_{0}\right)\right)$, there is a unique solution of (2.1), (2.3), (2.4) if and only if the operator $F_{3}$ is locally injective at $u_{0}$.

Moreover, if (C1) is assumed, then

(d) for each compact set of $Y_{3}$, the corresponding set of all solutions is compact (possibly empty).

Proof. Assertion (a) is evident by Lemmas 3.1 and 3.3.

Using the Nikolskii theorem for $A_{3}$, we can write

$$
F_{3}=C_{3}+\left(T_{3}+N_{3}\right)
$$

where $C_{3}: X_{3} \rightarrow Y_{3}$ is a linear homeomorphism and is proper (see [8, Proposition 2.1]) and $T_{3}+N_{3}: X_{3} \rightarrow Y_{3}$ is a completely continuous mapping.

Now take the compact sets $K \subset Y_{3}$ and $F_{3}^{-1}(K)$. Then there exists a sequence of the closed and bounded sets $M_{n} \subset F_{3}^{-1}(K) \subset X_{3}$ for $n=1,2, \ldots$ such that $\bigcup_{n=1}^{\infty} M_{n}=F_{3}^{-1}(K)$.

According to [8, Proposition 2.2], the restrictions $\left.F_{3}\right|_{M_{n}}$ for $n=1,2, \ldots$ are proper mappings and $\left[\left.F_{3}\right|_{M_{n}}\right]^{-1}(K)=M_{n}$ is a compact set. Hence, the operator $F_{3}$ is $\sigma$-proper, which gives the result (b).

Assertion (d) is a direct consequence of [8, Proposition 2.2].

Suppose now that $F_{3}$ is injective in a neighborhood $U\left(u_{0}\right)$ of $u_{0} \in X_{3}$. From the decomposition (4.1) the mapping

$$
C_{3}^{-1} F_{3}=I+C_{3}^{-1}\left(T_{3}+N_{3}\right)
$$

where $I: X \rightarrow Y$ is the identity, is completely continuous and injective in $U\left(u_{0}\right)$. On the basis of the Schauder domain invariance theorem (see [3, page 66]), the set $C_{3}^{-1} F_{3}\left(U\left(u_{0}\right)\right)$ is open in $X_{3}$ and the restriction $\left.C_{3}^{-1} F_{3}\right|_{U\left(u_{0}\right)}$ is a homeomorphism of $U\left(u_{0}\right)$ onto $C_{3}^{-1} F_{3}\left(U\left(u_{0}\right)\right)$. Therefore, $F_{3}$ is locally invertible. From Lemma 4.1 we obtain $(c)$.

The most important properties of the mapping $F_{3}$, whereby $A_{3}$ is a linear bounded Fredholm operator of zero index, $N_{3}$ is completely continuous, and $F_{3}$ is coercive, give the following theorem.

Theorem 4.4. If hypotheses (A1), (A2), (B1), (B3), and (C1) are satisfied, then for the initial-boundary value problem (2.1), (2.3), (2.4), the following statements hold.

(e) For each $g \in Y_{3}$, the set $S_{3 g}$ of all solutions is compact (possibly empty).

(f) The set $R\left(F_{3}\right)=\left\{g \in Y_{3}\right.$ : there exists at least one solution of the given problem $\}$ is closed and connected in $Y_{3}$.

(g) The domain of bifurcation $D_{3 b}$ is closed in $X_{3}$ and the bifurcation range $R_{3 b}$ is closed in $Y_{3} . F_{3}\left(X_{3}-D_{3 b}\right)$ is open in $Y_{3}$.

(h) If $Y_{3}-R_{3 b} \neq \varnothing$, then each component of $Y_{3}-R_{3 b}$ is a nonempty open set (i.e., a domain). 
The number $n_{3 g}$ of solutions is finite, constant (it may be zero) on each component of the set $Y_{3}-R_{3 b}$, that is, for every $g$ belonging to the same component of $Y_{3}-R_{3 b}$.

(i) If $R_{3 b}=0$, then the given problem has a unique solution $u \in X_{3}$ for each $g \in Y_{3}$ and this solution continuously depends on $g$ as a mapping from $Y_{3}$ onto $X_{3}$.

(j) If $R_{3 b} \neq \varnothing$, then the boundary of the $F_{3}$-image of the set of all points from $X_{3}$ in which the operator $F_{3}$ is locally invertible is a subset of the $F_{3}$-image of the set of all points from $\mathrm{X}_{3}$ in which $\mathrm{F}_{3}$ is not locally invertible, that is,

$$
\partial F_{3}\left(X_{3}-D_{3 b}\right) \subset F_{3}\left(D_{3 b}\right)=R_{3 b}
$$

Proof. Statement (e) follows immediately from Theorem 4.3(d).

(f) Let the sequence $\left\{g_{n}\right\}_{n \in \mathbb{N}} \subset R\left(F_{3}\right) \subset Y_{3}$ converge to $g \in Y_{3}$ as $n \rightarrow \infty$. By Theorem 4.3(d), there is a compact set of all solutions $\left\{u_{\gamma}\right\}_{\gamma \in I} \subset X_{3}$ ( $I$ is an index set) of the equations $F_{3}(u)=g_{n}$ for all $n=1,2, \ldots$. Then there exists a sequence $\left\{u_{n_{k}}\right\}_{k \in \mathbb{N}} \subset\left\{u_{\gamma}\right\}_{\gamma \in I}$ converging to $u \in X_{3}$ for which $F_{3}\left(u_{n_{k}}\right)=g_{n_{k}} \rightarrow g$. Since the operator $F_{3}$ is proper, whence it is closed, we have $F_{3}(u)=g$. Hence, $g \in R\left(F_{3}\right)$ and $R\left(F_{3}\right)$ is a closed set.

The connectedness of $R\left(F_{3}\right)=F_{3}\left(X_{3}\right)$ follows from the fact that $R\left(F_{3}\right)$ is a continuous image of the connected set $X_{3}$.

(g) According to Lemma 4.1(2), $D_{3 b}=\Sigma_{3}$ and $R_{3 b}=F_{3}\left(D_{3 b}\right)$. Since $X_{3}-\Sigma_{3}$ is an open set, $D_{3 b}$ and its continuous image $R_{3 b}$ are closed sets in $X_{3}$ and $Y_{3}$, respectively.

Since $X_{3}-D_{3 b}$ is a set of all points in which the mapping $F_{3}$ is locally invertible, then it ensures that to each $u_{0} \in X_{3}-D_{3 b}$ there is a neighborhood $U_{1}\left(F_{3}\left(u_{0}\right)\right) \subset F_{3}\left(X_{3}-D_{3 b}\right)$, which means that the set $F_{3}\left(X_{3}-D_{3 b}\right)$ is open.

(h) The set $Y_{3}-R_{3 b}=Y_{3}-F_{3}\left(D_{3 b}\right) \neq 0$ is open in $Y_{3}$, then each of its components is nonempty and open.

The second part of (h) follows from Ambrosetti theorem [1, page 216].

(i) Since $R_{3 b}=\varnothing$, the mapping $F_{3}$ is locally invertible in $X_{3}$. From [8, Proposition 2.2], we get that $F_{3}$ is a proper mapping. Then the global inverse mapping theorem [12, page 174] proves this statement.

(j) By (f) and (g), we have $\left(\Sigma_{3}=D_{3 b}\right)$

$$
F_{3}\left(X_{3}\right)=F_{3}\left(\Sigma_{3}\right) \cup F_{3}\left(X_{3}-\Sigma_{3}\right)=F_{3}\left(\Sigma_{3}\right) \cup \overline{F_{3}\left(X_{3}-\Sigma_{3}\right)}=\overline{F\left(X_{3}\right)} .
$$

Furthermore, $\partial F_{3}\left(X_{3}-\Sigma_{3}\right)=\overline{F\left(X_{3}-\Sigma_{3}\right)}-F\left(X_{3}-\Sigma_{3}\right)$, and thus the previous equality implies assertion $(\mathrm{j})$.

Theorem 4.5. Under assumption (A1), (A2), (B1), (B3), and (C1), each of the following conditions is sufficient for the solvability of problem (2.1), (2.3), (2.4) for each $g \in Y_{3}$ :

(k) for each $g \in R_{3 b}$, there is a solution $u$ of (2.1), (2.3), (2.4) such that $u \in X_{3}-D_{3 b}$;

(l) the set $Y_{3}-R_{3 b}$ is connected and there is a $g \in R\left(F_{3}\right)-R_{3 b}$.

Proof. First of all, we see that conditions (k) and (l) are mutually equivalent to the following conditions: 
422 Solutions of nonlinear initial-boundary value problems

$\left(\mathrm{k}^{\prime}\right) F_{3}\left(D_{3 b}\right) \subset F_{3}\left(X_{3}-D_{3 b}\right)$,

( $\left.\mathrm{l}^{\prime}\right) Y_{3}-R_{3 b}$ is a connected set and

$$
F_{3}\left(X_{3}-D_{3 b}\right)-R_{3 b} \neq \varnothing
$$

respectively $\left(D_{3 b}=\Sigma_{3}\right)$.

Then it is sufficient to show that conditions $\left(\mathrm{k}^{\prime}\right)$ and $\left(\mathrm{l}^{\prime}\right)$, respectively, are sufficient for the surjectivity of the operator $F_{3}: X_{3} \rightarrow Y_{3}$.

$\left(\mathrm{k}^{\prime}\right)$ From the first equality of $(4.4)$, we obtain $F_{3}\left(X_{3}\right)=F_{3}\left(X_{3}-D_{3 b}\right)$. Hence, $R\left(F_{3}\right)$ is an open as well as a closed subset of the connected space $Y_{3}$. Thus, $R\left(F_{3}\right)=Y_{3}$.

$\left(\mathrm{l}^{\prime}\right)$ By Theorem 4.4(h), $\operatorname{card} F_{3}^{-1}(\{q\})=$ const $=: k \geq 0$ for every $q \in Y_{3}-R_{3 b}$.

If $k=0$, then $F_{3}\left(X_{3}\right)=R_{3 b}$ and $F_{3}\left(X_{3}-D_{3 b}\right) \subset R_{3 b}$. This is a contradiction to (4.5). Then $k>0$ and $R\left(F_{3}\right)=Y_{3}$.

The other surjectivity theorem is true.

Theorem 4.6. Let hypotheses (A1), (A2), (B1), (B3), and (C1) hold and

(i) there exists a constant $K>0$ such that all solutions $u \in X_{3}$ of the initial-boundary value problem for the equation

$$
C_{3} u+\mu\left[A_{3} u-C_{3} u+N_{3} u\right]=0, \quad \mu \in(0,1),
$$

with data (2.3), (2.4), fulfil one of conditions $\left(\alpha_{1}\right)$ and $\left(\alpha_{2}\right)$ of the almost coercive condition (C1), then

(m) problem (2.1), (2.3), (2.4) has at least one solution for each $g \in Y_{3}$;

(n) the number $n_{3 g}$ of solutions of (2.1), (2.3), (2.4) is finite, constant, and different from zero on each component of the set $Y_{3}-R_{3 b}$ (for all $g$ belonging to the same component of $\left.Y_{3}-R_{3 b}\right)$.

Proof. (m) It is sufficient to prove the surjectivity of the mapping $F_{3}: X_{3} \rightarrow Y_{3}$. By Lemma 3.1, we can write

$$
F_{3}=A_{3}+N_{3}=C_{3}+\left(T_{3}+N_{3}\right)
$$

where $C_{3}: X_{3} \rightarrow Y_{3}$ is a linear homeomorphism from $X_{3}$ onto $Y_{3}$ and $T_{3}+N_{3}: X_{3} \rightarrow Y_{3}$ is a completely continuous operator. Then the operator

$$
C_{3}^{-1} F_{3}=I+C_{3}^{-1}\left(T_{3}+N_{3}\right): X_{3} \longrightarrow X_{3}
$$

is completely continuous and condensing (see [12, page 496]). The set $\Sigma_{3}=D_{3 b}$ is the set of all points $u \in X_{3}$ where $C_{3}^{-1} F_{3}$, as well as $F_{3}$, is not locally invertible.

Denote $S_{1} \subset X_{3}$ a bounded set. Then $C_{3}\left(S_{1}\right)=: S$ is bounded in $Y_{3}$, and by Lemma 3.4, $F_{3}^{-1}(S)=F_{3}^{-1}\left(C_{3}\left(S_{1}\right)\right)=\left(C_{3}^{-1} \circ F_{3}\right)^{-1}\left(S_{1}\right)$ is a bounded set in $X_{3}$. Thus, the operator $C_{3}^{-1} \circ$ $\mathrm{F}_{3}$ is coercive.

Now we show that condition (i) implies the conditions from [8, Theorem 3.2, Corollary 3.3, and Remark 3.1] for $F(u)=C_{3}^{-1} \circ F_{3}(u)$ and $C(u)=G(u)=u, u \in X_{3}$. 
In fact, as $C_{3}^{-1} \circ F_{3}(u)=k u$ if and only if $F_{3}(u)=k C_{3}(u)$, we get for $k<0$,

$$
C_{3} u+(1-k)^{-1}\left[A_{3} u-C_{3} u+N_{3} u\right]=0,
$$

where $(1-k)^{-1} \in(0,1)$.

In case $\left(\alpha_{1}\right)$, there is a constant $K>0$ such that for all solutions $u \in X_{3}$ of (4.9),

$$
\|u\|_{(1+\alpha) / 2,1+\alpha, Q} \leq K
$$

and in case $\left(\alpha_{2}\right)$,

$$
\|u\|_{(2+\alpha) / 2,2+\alpha, Q} \leq K
$$

Furthermore, by the same method as in Lemma 3.4, we get the estimation

$$
\|u\|_{X_{3}}<K_{1}, \quad K_{1}>0
$$

for all solutions $u \in X_{3}$ of $C_{3}^{-1} \circ F_{3} u=k u$. Hence, we get the surjectivity of $F_{3}$ and thus $(\mathrm{m})$.

(n) From Theorem 4.4(h) and the surjectivity of $F_{3}$, it follows that there is $n_{3 g} \neq 0$. This finishes the proof of Theorem 4.6.

\section{References}

[1] A. Ambrosetti, Global inversion theorems and applications to nonlinear problems, Confer. Sem. Mat. Univ. Bari (1979), no. 163-168, 211-232.

[2] L. Brüll and J. Mawhin, Finiteness of the set of solutions of some boundary-value problems for ordinary differential equations, Arch. Math. (Brno) 24 (1988), no. 4, 163-172.

[3] J. Dugundji and A. Granas, Fixed Point Theory. I, Monografie Matematyczne, vol. 61, Państwowe Wydawnictwo Naukowe (PWN), Warsaw, 1982.

[4] V. Ďurikovič, An initial-boundary value problem for quasi-linear parabolic systems of higher order, Ann. Polon. Math. 30 (1974), 145-164.

[5]_ Generic properties of the nonlinear mixed Dirichlet problem, Proceedings of the International Scientific Conference of Mathematics (Žilina, 1998), Žilina University Publisher, Žilina, 1999, pp. 57-63.

[6] V. Durikovič and Má. Ďurikovičová, Some generic properties of nonlinear second order diffusional type problem, Arch. Math. (Brno) 35 (1999), no. 3, 229-244.

[7] J. Mawhin, Generic properties of nonlinear boundary value problems, Differential Equations and Mathematical Physics (Birmingham, Ala, 1990), Math. Sci. Engrg., vol. 186, Academic Press, Massachusetts, 1992, pp. 217-234.

[8] V. Šeda, Fredholm mappings and the generalized boundary value problem, Differential Integral Equations 8 (1995), no. 1, 19-40.

[9] A. E. Taylor, Introduction to Functional Analysis, John Wiley \& Sons, New York, 1958.

[10] V. A. Trenogin, Functional Analysis, Nauka, Moscow, 1980.

[11] G. J. Šilov, Mathematical Analysis, Alfa-Vydavatelstvo Technickej a Ekonomickej Literatury, Bratislava, 1974 . 


\section{Solutions of nonlinear initial-boundary value problems}

[12] E. Zeidler, Nonlinear Functional Analysis and Its Applications. I. Fixed-Point Theorems, SpringerVerlag, New York, 1986.

Vladimír Ďurikovič: Department of Applied Mathematics, SS. Cyril and Methodius University, nám. J. Herdu 2, 91700 Trnava, Slovakia

E-mail address: vdurikovic@fmph.uniba.sk

Monika Ďurikovičová: Department of Mathematics, Slovak Technical University, nám. Slobody 17, 81231 Bratislava, Slovakia

E-mail address: durikovi@sjf.stuba.sk 


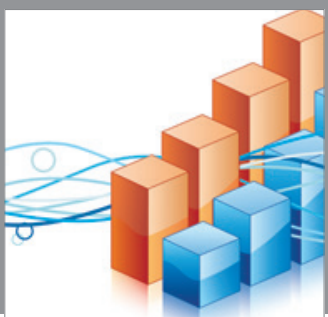

Advances in

Operations Research

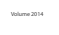

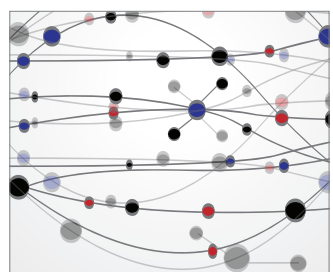

\section{The Scientific} World Journal
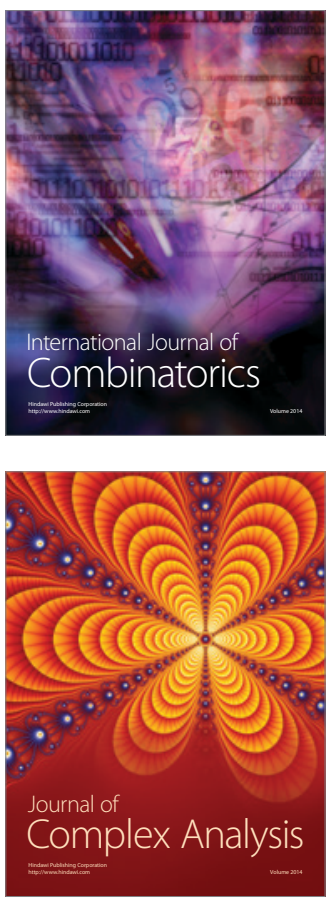

International Journal of

Mathematics and

Mathematical

Sciences
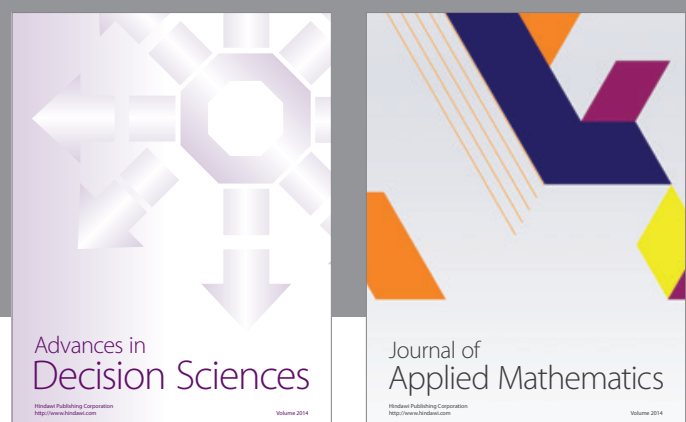

Journal of

Applied Mathematics
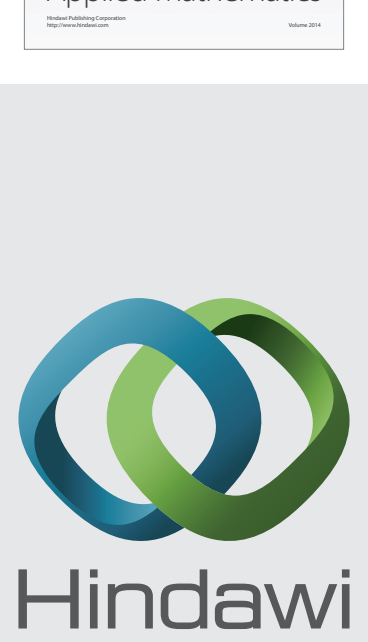

Submit your manuscripts at http://www.hindawi.com
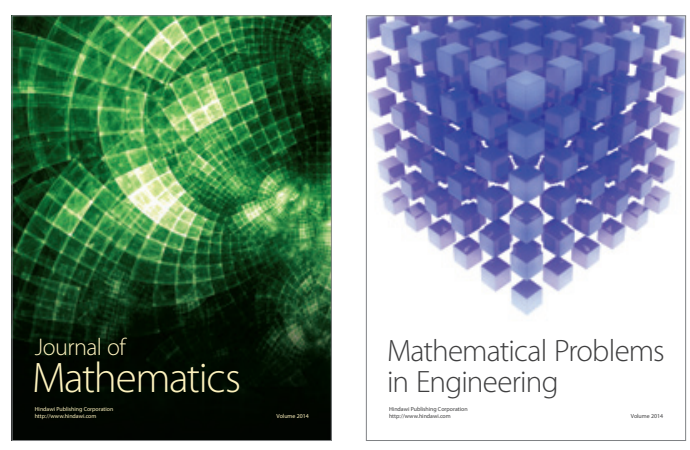

Mathematical Problems in Engineering
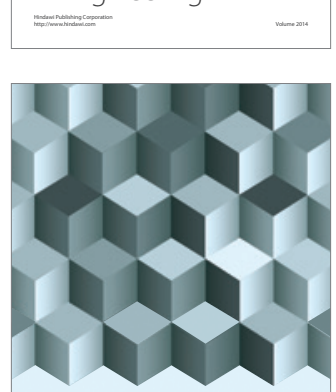

Journal of

Function Spaces
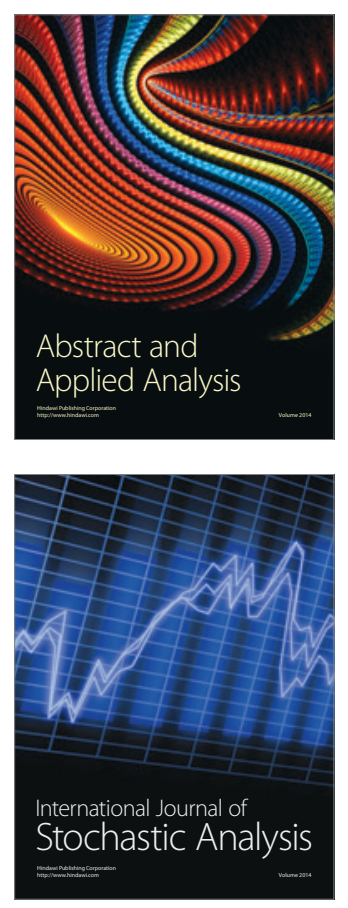

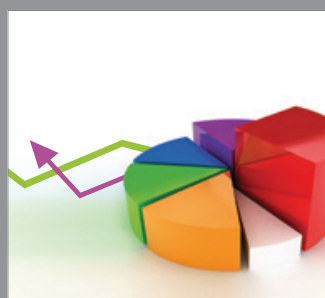

ournal of

Probability and Statistics

Promensencen
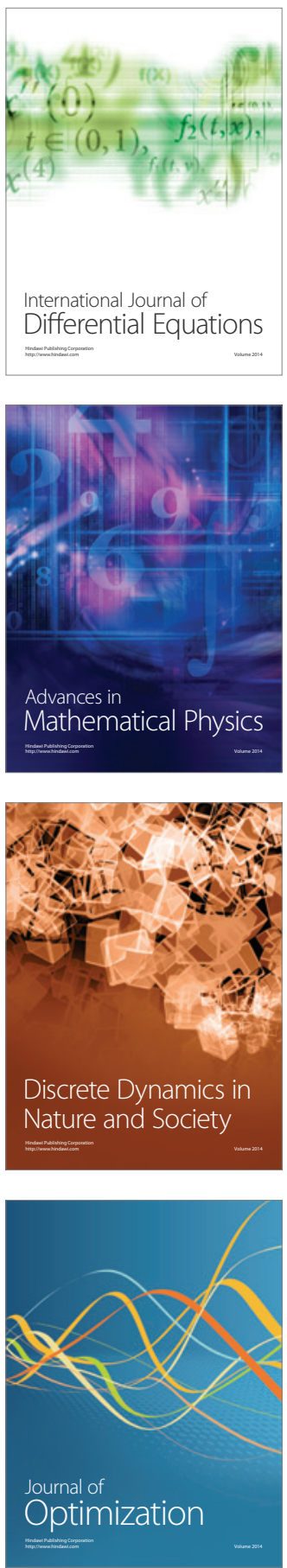